Tropical Journal of Pharmaceutical Research November 2017; 16 (11): 2713-2725

ISSN: $1596-5996$ (print); 1596-9827 (electronic)

(c) Pharmacotherapy Group, Faculty of Pharmacy, University of Benin, Benin City, 300001 Nigeria.

All rights reserved.

Available online at http://www.tjpr.org

Original Research Article

http://dx.doi.org/10.4314/tjpr.v16i11.20

\title{
Microscopic imaging ellipsometry of submicron-scale bacterial cells
}

\author{
Mai Ibrahim Khaleel ${ }^{1-3}$, Yu-Da Chen ${ }^{1-3}$, Ching-Hang Chien ${ }^{1-3}$ and Yia-Chung \\ Chang $^{1 *}$ \\ ${ }^{1}$ Research Center for Applied Sciences, Academia Sinica, Taipei, 11529, ${ }^{2}$ Nano Science and Technology Program, Taiwan \\ International Graduate Program, Academia Sinica and National Tsing Hua University, ${ }^{3}$ Department of Engineering and System \\ Science, National Tsing Hua University, Hsinchu, 30013, Taiwan
}

*For correspondence: Email: yiachang@gate.sinica.edu.tw

Sent for review: 27 July 2017

Revised accepted: 23 October 2017

\begin{abstract}
Purpose: To demonstrate the power of microscopic imaging ellipsometry (MIE) to identify submicronscale bacterial cells and track their surface topology variation.

Methods: Microscopic imaging ellipsometry with rotating compensator configuration was used to measure the ellipsometric spectra for dried submicron-scale Streptococcus mutans cells cultured on gold ( $A u)$ film for wavelengths $(\lambda)$ in the visible range $(\lambda=490-710) \mathrm{nm}$. The ellipsometry characteristic images, Psi ( $\Psi$ ), Delta $(\Delta), p$ - and s- polarized reflectance (Ip and Is), and reflectance difference image (RDI) for a chain of two and four dried cells, were collected for a series of different objective planes (near the focal plane) for $\lambda=600 \mathrm{~nm}$.

Results: The results show that by adjusting the position of objective planes (POP) to achieve the best focus, it was possible to identify cells smaller than $1 \mu \mathrm{m}$ and observe their diffraction patterns in $\psi$ and $\Delta$ images. It was observed that $\Delta$ spectra and images were particularly sensitive to POP, while $\psi$ spectra and images for dried $S$. mutans cells were rather insensitive to POP.

Conclusion: MIE is a sensitive non-optical technique that can be used to image biological systems without the need for labeling of molecules.
\end{abstract}

Keywords: Objective plane, Imaging ellipsometry, Submicron-scale bacterial cells, Streptococcus mutans, Biosensing, Focal plane

Tropical Journal of Pharmaceutical Research is indexed by Science Citation Index (SciSearch), Scopus, International Pharmaceutical Abstract, Chemical Abstracts, Embase, Index Copernicus, EBSCO, African Index Medicus, JournalSeek, Journal Citation Reports/Science Edition, Directory of Open Access Journals (DOAJ), African Journal Online, Bioline International, Open-J-Gate and Pharmacy Abstracts

\section{INTRODUCTION}

Ellipsometry is an optical technique used to determine the thickness and optical constants of samples based on measuring two characteristic parameters, $\psi$ (Psi) and $\Delta$ (Delta), which describe the polarization contrast and phase change of light reflected from the sample [2]. The parameters $\psi$ and $\Delta$ represent the amplitude ratio and phase difference between $p$ - and $s$ polarized light waves, which are related to the complex Fresnel reflection coefficients according to Eq 1. [2-4].

$\rho=\frac{{ }^{r} p}{r_{s}}=\tan \psi e^{-i \Delta}$

Throughout this paper, adopting the notations in physics community where the complex dielectric constant is defined as $\varepsilon=\varepsilon_{1}+i \varepsilon_{2}=(n+i k)^{2}$ with $n$ and $k$ being the refractive index and extinction coefficient, respectively. We define the phase $\Delta$ 
as given in Eq 1 instead of as used in the optics community. With this notation, the sign convention of $\Delta$ in both optics and physics communities become consistent [5].

Microscopic Imaging Ellipsometry (MIE) combines the capabilities of ellipsometry in measuring thin film thickness accurately with the high spatial resolution of optical microscopy in lateral directions [6]. The technique is well known for being non-contact, non-destructive, and noninvasive [7-9]. It does not require special sample preparation nor labeling of molecules, in comparison to other detection techniques that require labeling- usually enzymatic or fluorescent such as fluorescence imaging [9-11]. Comparing MIE to other non-invasive and label-free imaging techniques like IR/thermal imaging [12], and Raman spectroscopy [13]. MIE provides submicron spatial resolution that cannot be achieved by $\mathrm{IR} /$ thermal imaging technique since it is based on detecting thermal radiation from the sample and not capable of extracting highresolution structure information as MIE does [14]. Raman spectroscopy and MIE provide different but complementary information of the sample. MIE can detect very thin layers and measure their precise thickness, while Raman spectroscopy is sensitive to the chemical nature of the sample [13].

Streptococcus mutans (S. mutans) is an aerobic bacteria with a size $\sim 800 \mathrm{~nm}$ and average height equals to $350 \mathrm{~nm}$ [14]. It has been known as the main etiological agent of dental caries and normal inhabitant of dental plaque [14]. In a previous work, we proposed microscopic imaging ellipsometry with rotating compensator configuration (MIE-RCE) as a sensing tool to detect the distribution of dried $S$. mutans cells on a glass substrate and determine their optical constants and thickness using theoretical models based on effective medium theory [14]. In this paper, we present MIE-RCE measurements of a chain of two and four dried $S$. mutans cells on Au film in specular reflection mode for wavelengths $(\lambda)$ in the visible range $(\lambda=490 \mathrm{~nm}-710 \mathrm{~nm})$ on different objective planes. First, the MIE setup was calibrated for Au film, then the ellipsometry spectra of dried bacterial cells for different objective planes were measured at an angle of incident $\left(\mathrm{AOI}=52^{\circ}\right)$. A series of images for a chain of two and four dried $S$. mutans cells were captured for several objective planes, and images of $\psi, \Delta$, reflectance $\left(I_{s}=\left|r_{s}\right|^{2}\right), \mathrm{p}$ polarized reflectance $\quad\left(I_{p}=\left|r_{p}\right|^{2}\right)$, and reflectance difference image $\left(R D I=I_{s}-I_{p}\right)$ at $600 \mathrm{~nm}$ were drawn by using the Intel Array Viewer Software [15]. Performing measurements at different objective planes were done by moving the sample vertically via a motorized $z$ translation piezo stage (Physik Instrumente GmbH \& Co. KG, Germany) [16].

The MIE images presented in this work of the two and four dried $S$. mutans cells are divided into intensity related images $\left(I_{p}\right.$ and $\left.I_{s}\right)$, polarization-contrast related images ( $\psi$ and $R D I$ ), and phase related image $(\Delta)$ [17]. The intensity related images show the diffraction pattern outside the cells, the polarization-contrast related images give more information regarding the morphology of the cells, and the phase related image is sensitive to the refractive index and absorption coefficient of the cells. This study illustrates the versatility and the promise of MIE for future applications especially in the field of biological imaging since it enables us to identify submicron features, perform real-time measurements, and track the changes in biological systems without labeling the molecules.

\section{EXPERIMENTAL}

\section{Cell culture and sample preparation}

An S. mutans strain (ATCC 25175) and brainheart infusion broth $(\mathrm{BHI})$ were purchased from Creative Life Sciences (CMP), Taipei, Taiwan [18]. S. mutans was incubated in BHI medium shaken at $37 \stackrel{\circ}{\circ}$ overnight. Another solution consists of $7.4 \mathrm{~g} \mathrm{BHI}, 20 \mathrm{~g}$ sucrose, and $200 \mathrm{ml}$ Millipore water was prepared to grow bacterial cells on Au film [19]. A substrate consists of $\mathrm{SiO}_{2}$ $300 \mathrm{~nm} / \mathrm{Ti} 40 \mathrm{~nm} / \mathrm{Au} 50 \mathrm{~nm}$ was cleaned by sonication in acetone and isopropanol solutions for 30 and 20 minutes respectively. After that, the substrate was sonicated in Millipore water for 10 minutes and finally dried in the oven.

S. mutans was cultured on the Au film by immersing the cleaned substrate into a Petri dish contains $10 \mathrm{ml}$ of BHI-sucrose solution and $2.5 \mathrm{ml}$ of incubated S. mutans cells in $\mathrm{BHI}$ medium. The Petri dish was sealed with parafilm and incubated for $6 \mathrm{~h}$ at $37^{\circ} \mathrm{C}$. After that, the substrate was washed three times with Millipore water and dried at room temperature for one day [14].

\section{MIE-RCE setup}

In this study, Optrel Multiskop system [20] , was used to do the measurements. It based on the concept of rotating compensator ellipsometry. It consists of three main components: laser arm, sample stage, and detector arm as shown in Figure 1 [14]. The light source is generated from 
a Fianium WhiteLase short wavelength supercontinuum laser with power $>300 \mathrm{~mW}$ and wavelength from $400 \mathrm{~nm}$ to $1000 \mathrm{~nm}$ [21]. The light beam then guided into a low-loss fiber connected to the laser arm of the system where a polarizer (Glan-Thompson Polarizing Prism, Germany) [22], is fixed at 45․ A rotating compensator is added to the laser arm and monitored by a goniometer. The compensator is a quarter-wave plate and rotates with the range of angles from $5^{\circ}$ to $355^{\circ}$ with $10^{\circ}$ steps. The intensity of the incident light beam with spot size around $100 \mu \mathrm{m}$ is enhanced by using a $10 \mathrm{X}$ lens before it hits the spot of interest on the sample.

The scattered or reflected light from the sample is collected by an $80 \mathrm{X}$ objective lens with numerical aperture equals to 0.5 , attached to the detector arm [23]. After that, the light beam passes through an analyzer mounted at $90^{\circ}$ with respect to the polarizer [14]. Finally, the light beam is collected by a charge coupled device
(CCD) camera (Pixelfly qe) [24], with a pixel size equal to $164 \mathrm{~nm}$.

The intensity of the reflected light beam from the sample collected by the CCD camera is then analyzed to determine the ellipsometric spectra and images. The sample stage of MIE-RCE consists of a motorized z-translation piezo stage fixed on a manual stage as shown in Figure 2. The motorized piezo stage is controlled by LabView program with $10 \mathrm{~nm}$ spatial resolution in the $z$-direction for fine adjustment. The manual stage has two fine adjustment controls for $x$ - and $y$-directions with $10 \mu \mathrm{m}$ steps, and one coarse adjustment control for z-translation with $1 \mathrm{~mm}$ spatial resolution [17]. The fine resolution in the z-direction is used for fine tuning of the objective plane $P 1$ to $P 4$ in Fig. 2 where $P 1$ denotes the starting position of sample plane during the measurements, and by moving the sample stage up the objective planes reach $P 2, P 3$, and $P 4$.

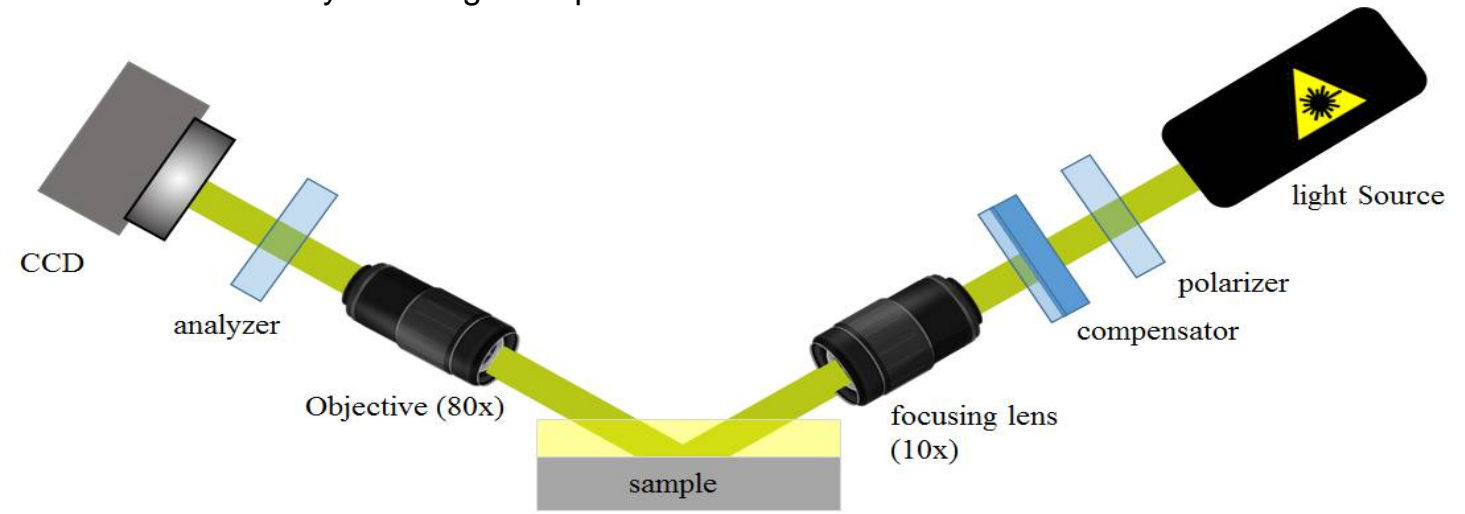

Figure 1: Schematic diagram of MIC RCE [14]

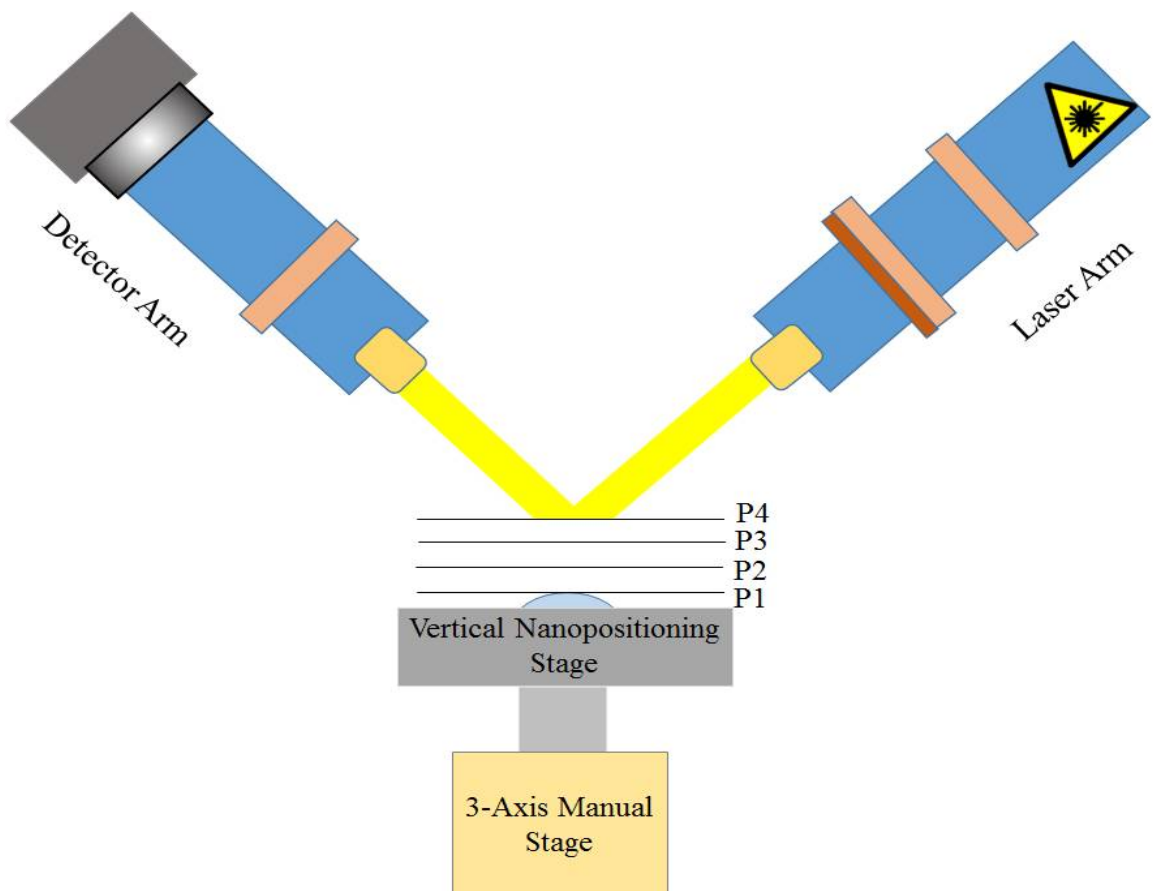

Figure 2: Schematic diagram of experimental setup using specular mode with different objective planes. 


\section{MIE-RCE calibration}

To measure the ellipsometry spectra and images of any sample, MIE-RCE system must be calibrated first by using a flat substrate like glass, $\mathrm{Si}$, or $\mathrm{Au}$. For our calibration process, we used $\mathrm{Si}$ substrate, and another sample consists of glass $/ \mathrm{Ti} 5 \mathrm{~nm} / \mathrm{Au} 50 \mathrm{~nm}$ to calibrate the system. To make sure that we obtained the correct sign for $\Delta$, we first measured the reflected light intensity of $\mathrm{Si}$ substrate and Au film as a function of compensator angle $I\left(\phi_{c}\right)$ in the visible range $(\lambda$ $=500 \mathrm{~nm}$ to $700 \mathrm{~nm}$ ) integrated over an area equals to $20 \mu \mathrm{m} \times 20 \mu \mathrm{m}$. For Si substrate and $\mathrm{Au}$ film, we performed measurements for $\mathrm{AOls}\left(72^{\circ}\right.$, $\left.82^{\circ}\right)$ and $\left(52^{\circ}, 58^{\circ}\right)$ respectively. The measured $\psi$ and $\Delta$ spectra for Si substrate and Au film by MIE-RCE were compared with $\psi$ and $\Delta$ spectra of the same $\mathrm{Si}$ substrate and Au film obtained by commercial V-VASE (J.A Woollam Co., United States) [25], for the same range of wavelengths and AOls. For Si and Au film, we can use the single interface model to calculate the Fresnel reflection coefficients $r_{p}$ and $r_{s}$ for $p$ - and $s$ polarized light [14], since $\mathrm{Si}$ and $\mathrm{Au}$ are opaque for the wavelength range considered here. We obtained

$$
\begin{gathered}
r_{p}=\frac{\frac{1}{c_{0}} \sqrt{\varepsilon_{0}-\sin \theta_{0}^{2}-\frac{1}{c_{1}} \sqrt{\varepsilon_{1}-\sin \theta_{0}^{2}}}}{\frac{1}{c_{0}} \sqrt{\varepsilon_{0}-\sin \theta_{0}^{2}+\frac{1}{c_{1}} \sqrt{\varepsilon_{1}-\sin \theta_{0}^{2}}}} \\
r_{s}=\frac{\sqrt{\varepsilon_{0}-\sin \theta_{0}^{2}-\sqrt{\varepsilon_{1}-\sin \theta_{0}^{2}}}}{\sqrt{\varepsilon_{0}-\sin \theta_{0}^{2}+\sqrt{\varepsilon_{1}-\sin \theta_{0}^{2}}}}
\end{gathered}
$$

where $\theta_{0}$ is the AOI, $\varepsilon_{0}$ and $\varepsilon_{1}=(n+i k)^{2}$ are the dielectric constant of ambient (air) and $\mathrm{Si}$ or $\mathrm{Au}$, respectively. Eqs. (2) and (3) was used to compute $\psi$ and $\Delta$ spectra for $\mathrm{Si}$ and Au film then compare with measured results obtained from $\mathrm{V}$ VASE and MIE-RCE. In Figure $3 a$ we show values of optical constants $n$ and $k$ for $\mathrm{Si}$ substrate obtained by V-VASE fitting software, which fit $\psi$ and $\Delta$ spectra. While in Figure $3 \mathrm{~b}$ we show values of optical constants $n$ and $k$ for $\mathrm{Au}$ film obtained by V-VASE fitting software, which fit $\psi$ and $\Delta$ spectra. Note that the fit takes into account the presence of $2 \mathrm{~nm}$ thick $\mathrm{SiO}_{2}$ on the $\mathrm{Si}$ surface, although its effect is negligible for the wavelength range $(500 \mathrm{~nm}-700 \mathrm{~nm})$ considered here. These values were used to calculate $r_{p}$ and $r_{s}$ (Eqs. (2) and (3)) for Si and Au film then obtain the theoretical values of $\psi$ and $\Delta$ without including the effect of oxide layer.

Figure 4 (a, b, c and d) show the comparison of $\psi$ and $\Delta$ spectra obtained by MIE-RCE, V-VASE, and theory for $\mathrm{Si}$ and $\mathrm{Au}$ film respectively. Figure 4 shows that both $\psi$ and $\Delta$ spectra of $\mathrm{Si}$ and $\mathrm{Au}$ film obtained by MIE-RCE, V-VASE, and theory (based on the single interface model) match well with each other in the full range of $\lambda$ from $500 \mathrm{~nm}$ to $700 \mathrm{~nm}$, indicating that the sign convention adopted in Eq 1 gives consistent results between our MIE-RCE setup and V-VASE.

\section{RESULTS}

Ellipsometry spectra and images were measured for two and four dried $S$. mutans cells cultured on Au film at different objective planes in specular reflection mode for $\lambda=(490-710 \mathrm{~nm})$ at an $\mathrm{AOI}$ $=52^{\circ}$. The ellipsometry characteristic images $\psi$, $\Delta$, p- and s- polarized reflectance $\left(I_{p}\right.$ and $\left.I_{s}\right)$, and reflectance difference image (RDI) for the two and four dried cells were collected for a series of different objective planes and images at $600 \mathrm{~nm}$. To capture the images of the $S$. mutans cells, we used a CCD camera with pixel size equivalent to $82 \mathrm{~nm}$ under the 80X magnification mounted at detector arm of our Multiskop setup as shown in Figure 1 previously. To reduce the statistical error, we combine every $2 \times 2$ grids to form a pixel of $164 \times 164 \mathrm{~nm}$, which gives more stable signal and better quality of MIE image. Both the

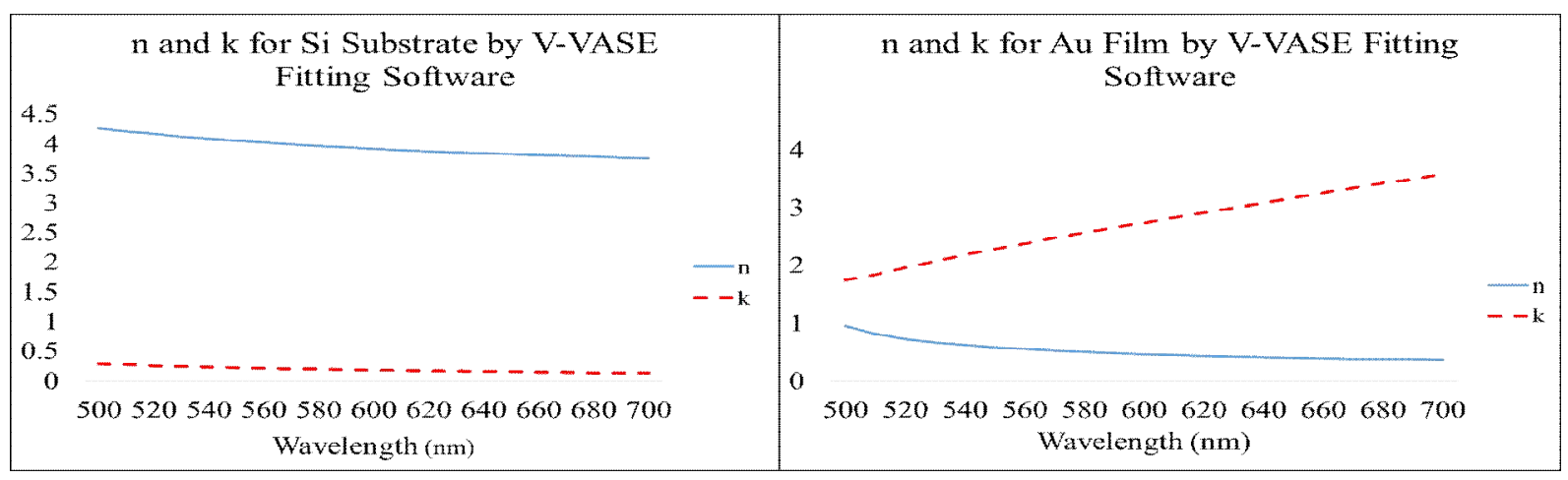

Figure 3: Optical constants $n$ and $k$ for (a) Si substrate, and (b) Au film obtained by V-VASE fitting software 


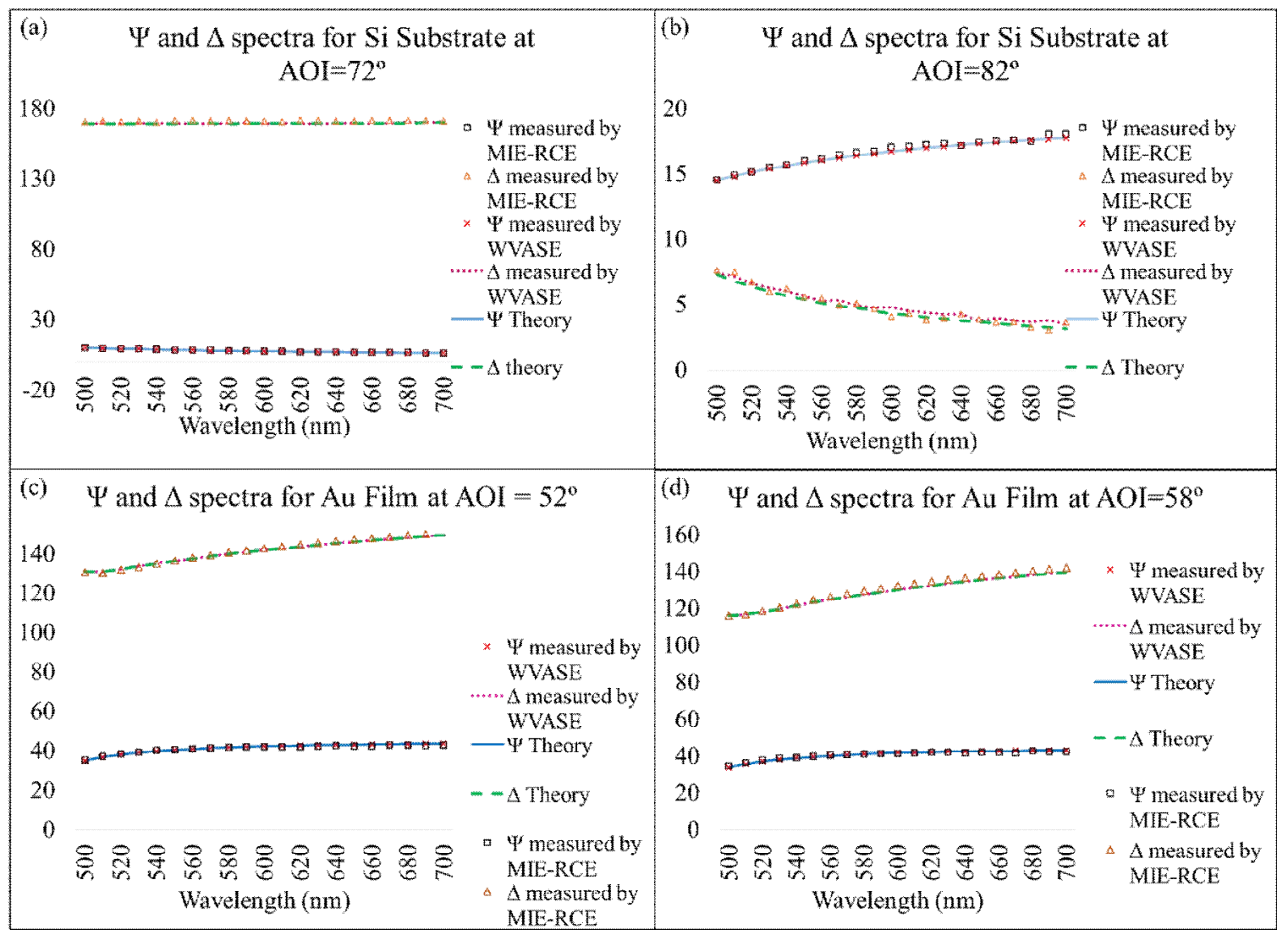

Figure 4: (a) $\psi$ and $\Delta$ for Si substrate obtained by MIE-RCE, V-VASE, and theory at $\mathrm{AOI}=72^{\circ}$, (b) $\psi$ and $\Delta$ for Si substrate obtained by MIE-RCE, V-VASE, and theory at $\mathrm{AOI}=82^{\circ}$, (c) $\psi$ and $\Delta$ for Au film obtained by MIERCE, VVASE, and theory at $A O I=52^{\circ}$, (d) $\psi$ and $\Delta$ for Au film obtained by MIE-RCE, V-VASE, and theory at $\mathrm{AOI}=58^{\circ}$

top view images of $\Delta$ in the $x-y$ plane and the side view images of $-\Delta$ (from the detector arm) are shown to gain a better understanding. The units of the horizontal axis $(y)$ in all the side view images of two and four dried $S$. mutans cells are pixels (one pixel $=164 \mathrm{~nm}$ ).

\section{MIE images for different objective planes of two S. mutans cells on Au film}

The ellipsometry images $\Psi, \Delta, I_{p}, I_{s}$, and $R D I$ for two dried $S$. mutans cells on Au film collected at seventeen different objective planes are shown in Figure $5(P 1-P 8)$ and $\mathrm{b}(P 9-P 17)$. The position of starting objective plane $P 1$ is labeled 0 , which is below the focal plane by several microns. We then moved up the sample with the motorized z-translation piezo stage. The advancement from the previous objective plane is labeled by $+Z$, where $z$ is the amount of movement in $\mathrm{nm}$ or $\mu \mathrm{m}$. At objective planes $P 1$ and $P 2$ the two cells are still way out of focus, and the $\Delta$ image in Fig. 5 shows variation with magnitude of $\Delta$ varying within $\pm 40^{\circ}$ from a flat background (which is around $140^{\circ}$ ) as seen in the side-view of $-\Delta$ image.
After moving the sample up $200 \mathrm{~nm}(P 3) \Delta$ image begins to show a noticeable change in the middle of the two cells which appears as a small hole in the top view of $\Delta$ image. The side view of $-\Delta$ image shows a sharp peak at that position with the magnitude of $-\Delta$ deviate from the flat background more than $300^{\circ}$. This is caused by the interference effect of two diffraction fringes arising from the two cells, which becomes significant as the objective plane approaches the focal plane. The side-view $-\triangle$ images at $P 4, P 5$, and $P 6$ show the increase in width of the sharp peak. At $P 7$ and $P 8$, the two cells became in focus (see CCD images), and $\Delta$ images show strong signals along the curved edge on the right side of the two cells, while $I_{p}$, and $I_{s}$ images of the same objective planes show two small spheres in the middle which are related to the two cells. It is noted that the width of the wall-like feature of the side-view $-\Delta$ image (along the $y$ axis) correlates directly with the length of the two cells when the objective plane coincides with the focal plane. It appears that the $\Delta$ image behaves like the gradient of the $\psi$ image, thus providing more sensitive detection of the boundary of the structure feature and it strongly depends on the position of the objective plane (POP). 


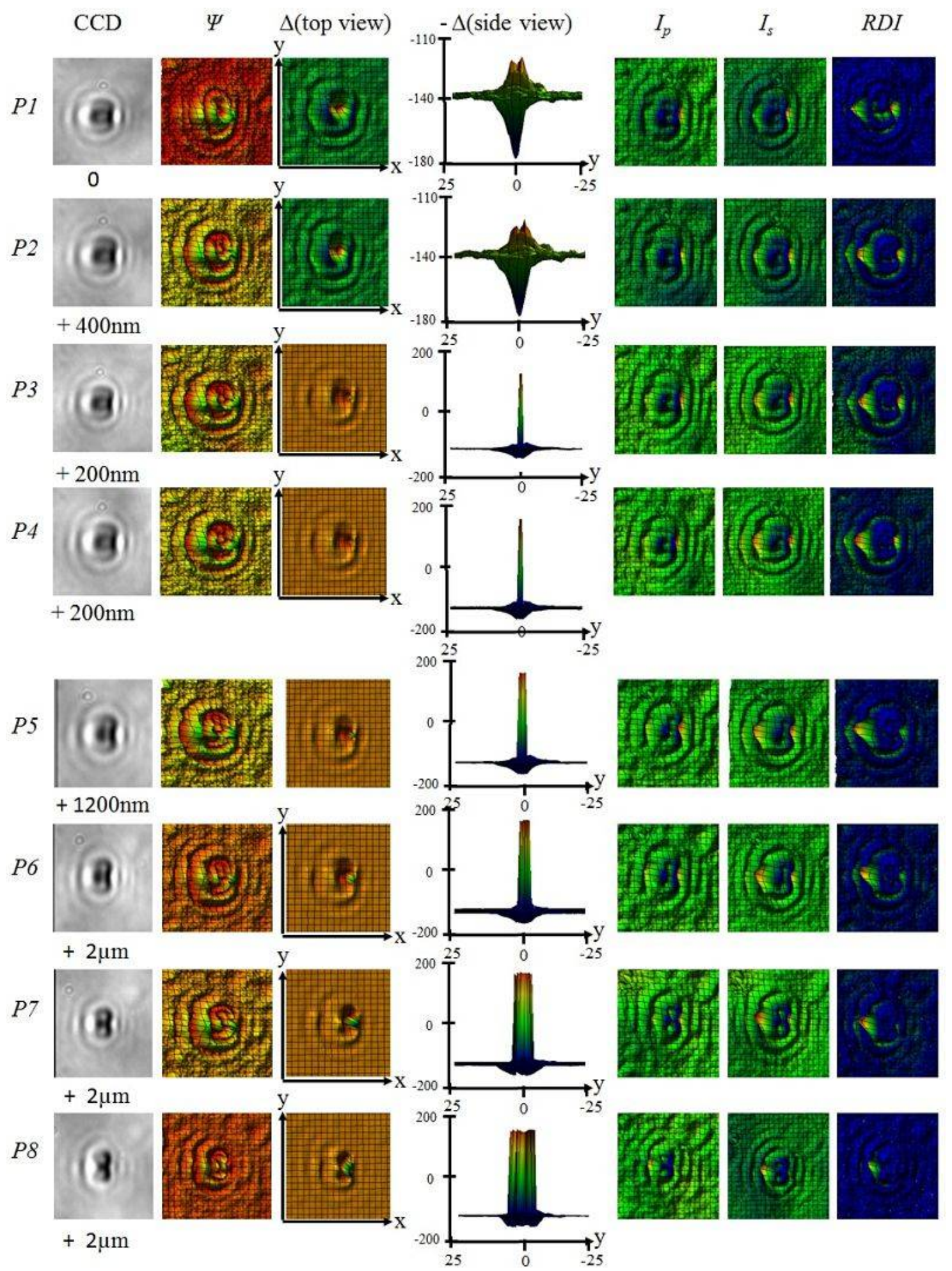

Figure 5: Ellipsometry images $\psi, \Delta, I_{p}, I_{s}$, and $R D I$ of two $S$. mutans cells on Au film for different objective planes $(P 91-P 8)$ at $\mathrm{AOI}=52^{\circ}$

In Figure 6 , the objective planes $P 9, P 10$, and $P 11$ are above the focal plane, and the light penetrates through the two cells and gets reflected at the interface between the cells and the Au film. At $P 10$ and $P 11$, the top-view $\Delta$ images show strong signals around the curved edges of the two cells and the side-view images of $-\Delta$ show a widened wall-like structure which is related to the diffraction fringes of the two cells. Moving the stage further up from P11 leads to noticeable distortion in the curved edge (at P12 and $P 13$ ), and the $\Delta$ signal became weaker and weaker as the stage moves up. (See side-view $\triangle$ images at $P 15-P 17$ ).

Trop J Pharm Res, November 2017; 16(11): 2718 


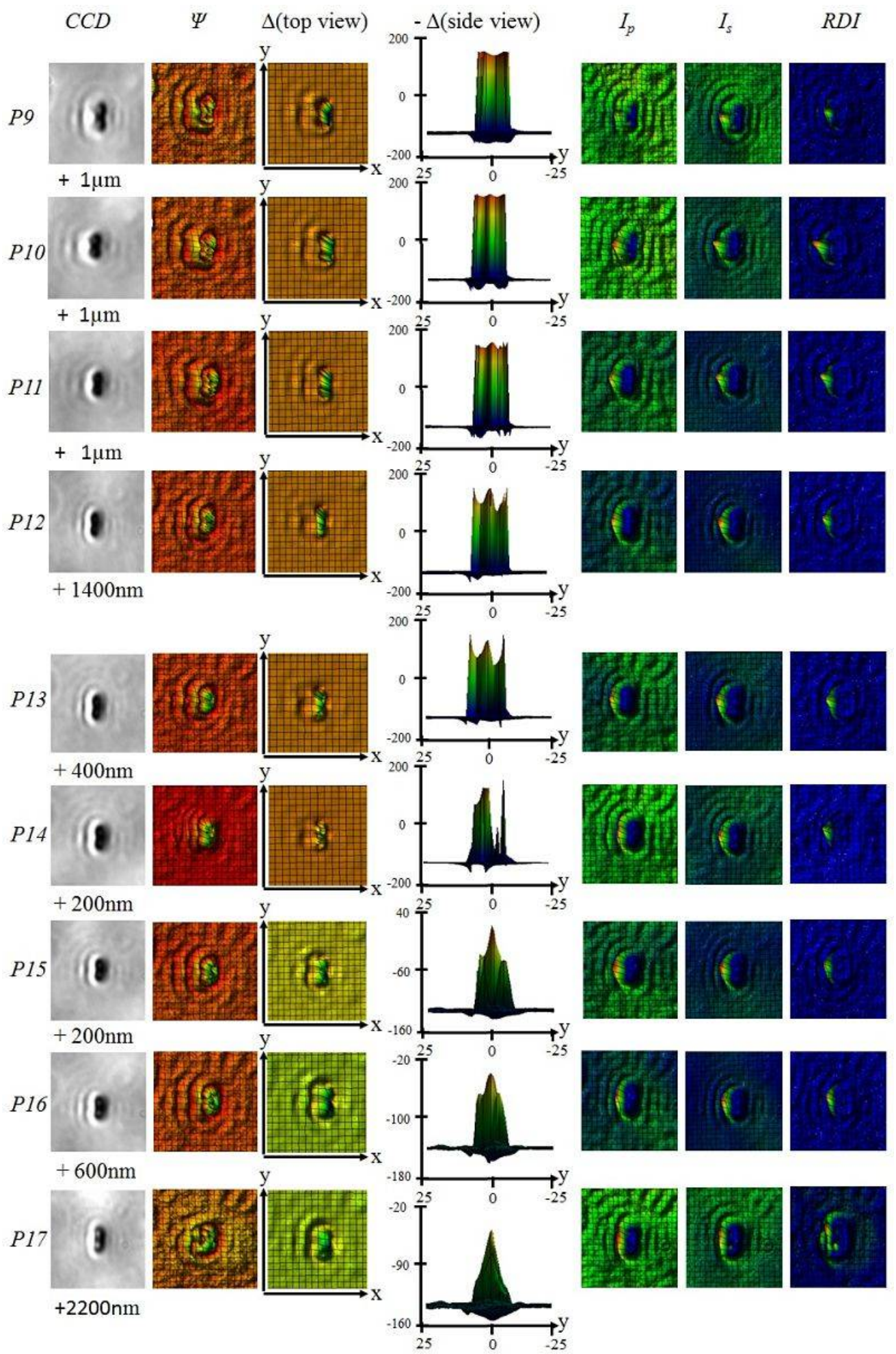

Figure 6: Ellipsometry images $\psi, \Delta, I_{D}, I_{s}$, and $R D I$ of two $S$. mutans cells on Au film for different objective planes $(P 9-P 17)$ at $\mathrm{AOI}=52^{\circ}$

\section{MIE images for different objective planes of four S. mutans cells on Au film}

To illustrate that the sensitivity of $\Delta$ image to the POP for the two-cell case is not coincidental, we show the ellipsometry images $\psi, \Delta, I_{p}, I_{s}$, and $R D I$ for a chain of four dried $S$. mutans cells on
Au film collected at nineteenth different objective planes are shown in Figs. $7(P 1-P 8), 8(P 9-$ $P 16)$, and Figure $9(P 17-P 19)$. At $P 1$, the four cells are way out of focus, and the $\Delta$ image shown in Figure 7 has weak variation (similar to $P 1$ in Fig. 5). After moving the sample up $200 \mathrm{~nm}$ to $P 2$, the $\Delta$ image exhibits significant change

Trop J Pharm Res, November 2017; 16(11): 2719 
near the first (lowest) cell of the four which appears as a sharp peak in the side view of $-\Delta$ image. Moving the stage up by $1800 \mathrm{~nm}$ to $P 3$, we see a widened peak associated with the first cell in the side-view of $-\Delta$ image. Because the chain of four cells is curved with the lower end tilt toward the incident arm, only the $\Delta$ image of the first cell shows the significant change, since it is closest to the incident arm. At P4 a second hole appeared at the last (highest) cell of the four in the top-view $\Delta$ image and the side-view of $-\Delta$ image shows a sharp peak, and the peak widened somewhat as the stage moved up slightly (by $600 \mathrm{~nm}$ ) to $P 5$. At $P 6$ a third hole (sharp peak) appeared in the top view of $\Delta$ image (side view of $-\Delta$ image), while at $P 7$ and $P 8$ the widths of all three peaks in the side view increased.

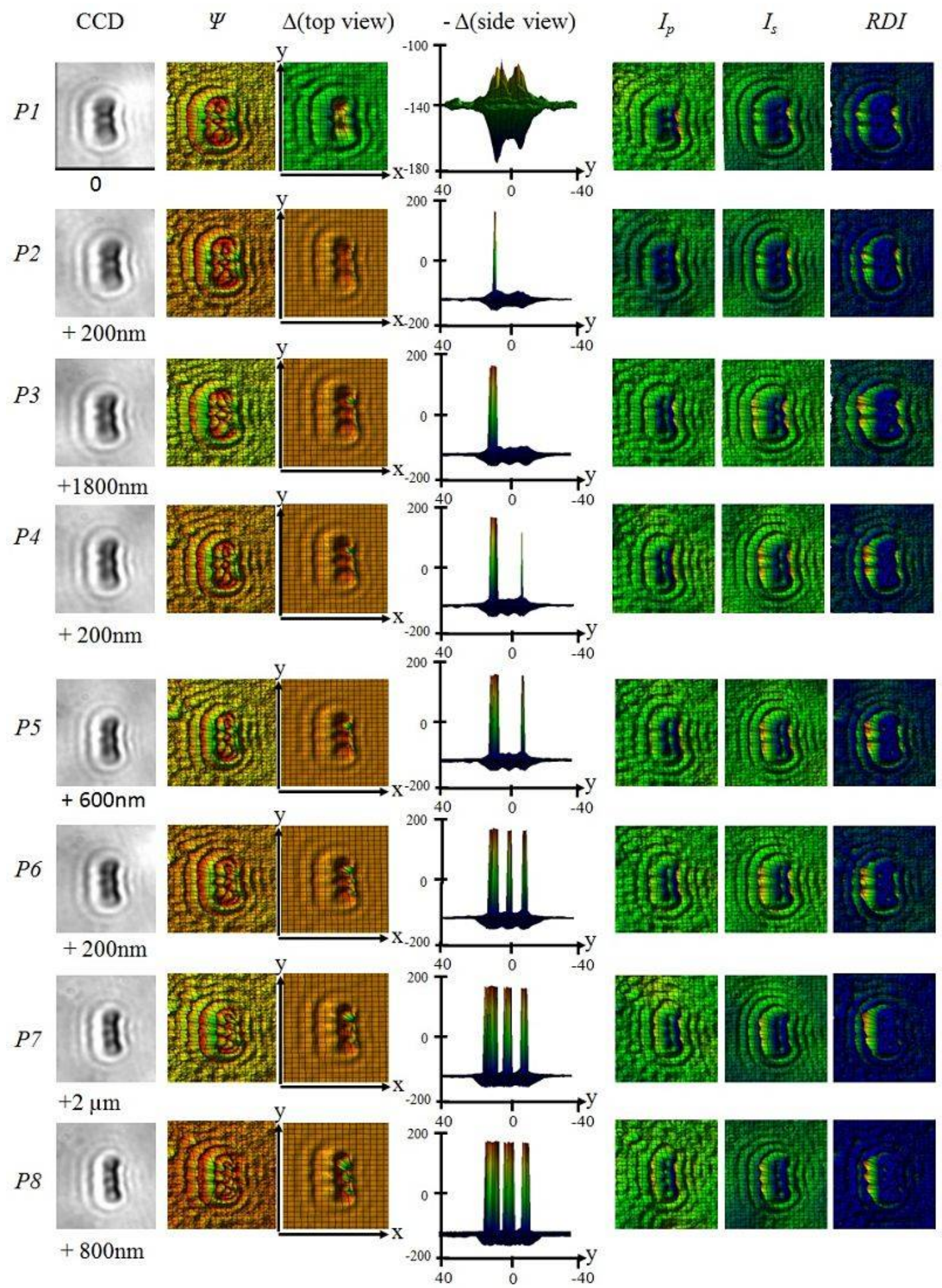

Figure 7: Ellipsometry images $\psi, \Delta, I_{p}, I_{s}$, and $R D I$ of four $S$. mutans cells on Au film for different objective planes $(P 1-P 8)$ at $\mathrm{AOI}=52^{\circ}$ 


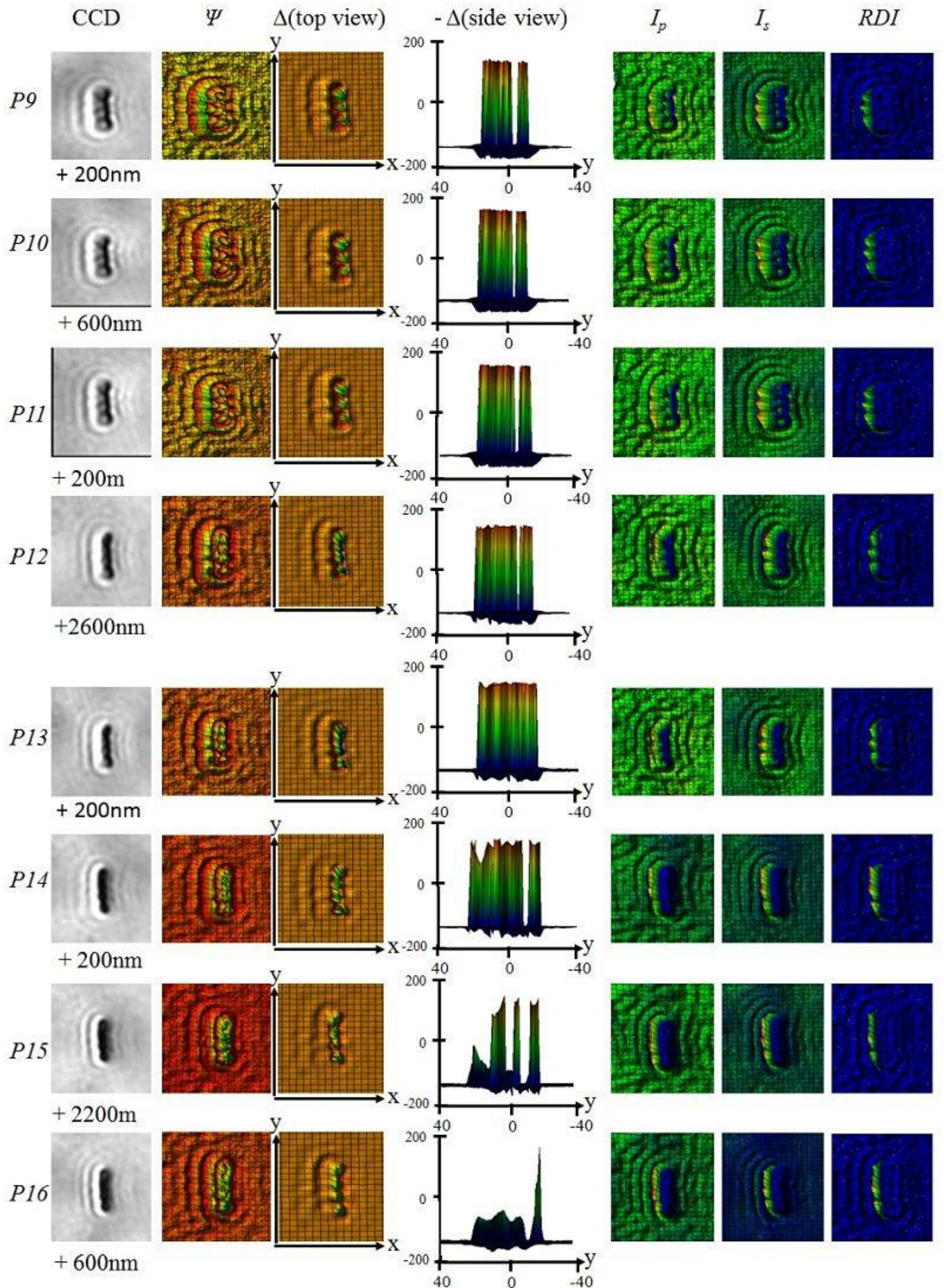

Figure 8: Ellipsometry images $\psi, \Delta, I_{p}, I_{s}$, and $R D I$ of four $S$. mutans cells on Au film for different objective planes $(P 9-P 16)$ at $\mathrm{AOI}=52^{\circ}$

We noticed that the width of the first peak (in the side view of $-\Delta$ image) is almost twice that of the second and third peaks, which is due to the newly picked-up signal from the second cell of the four. This conclusion is supported by the
CCD images and (the intensity related images $I_{p}$, $I_{s}$ ) at $P 7$ and $P 8$ that show a chain of four small spheres in the middle, which are related to four $S$. mutans cells. 

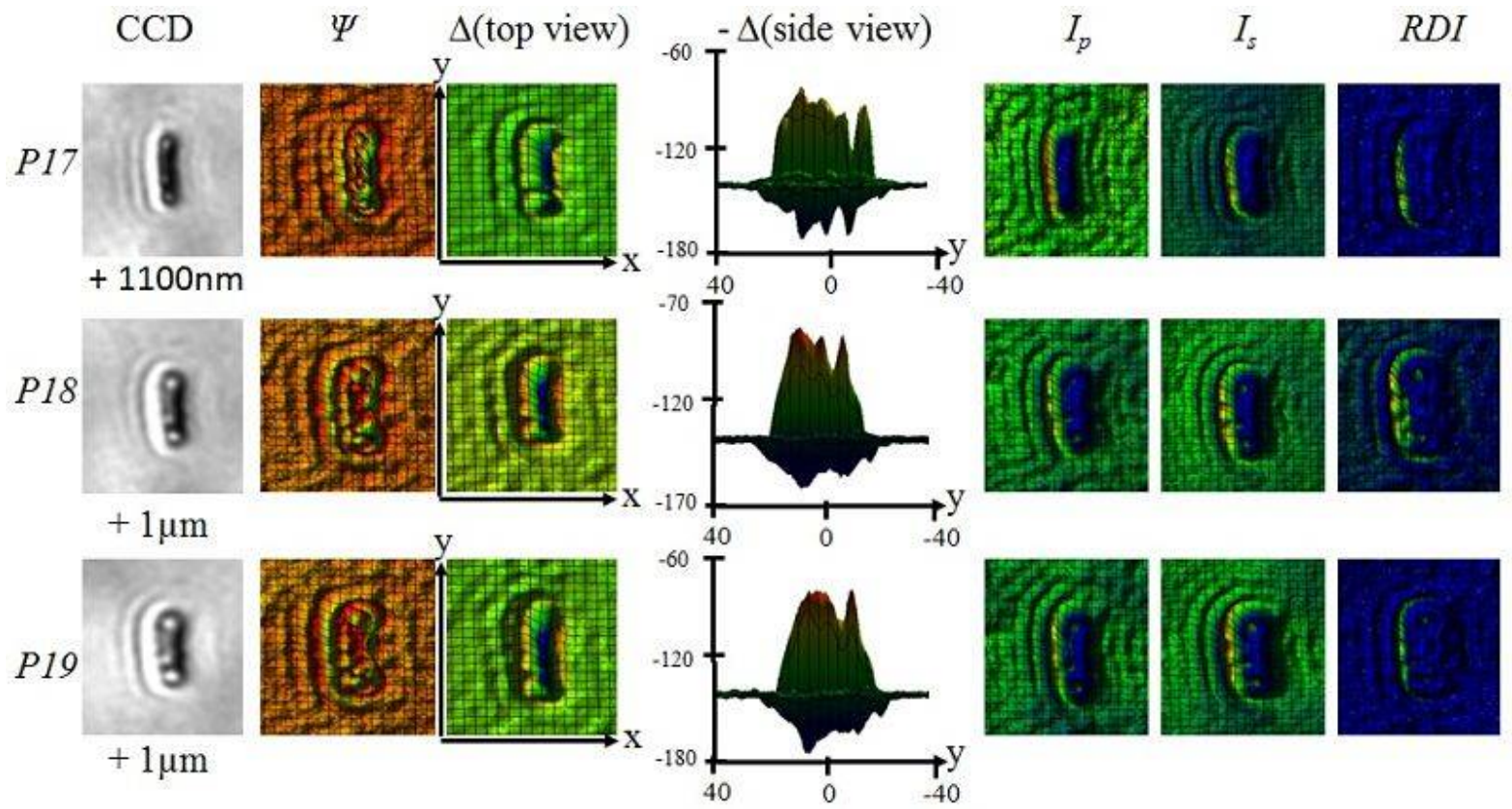

Figure 9: Ellipsometry images $\Psi, \Delta$, Ip, Is, and RDI of four $S$. mutans cells on Au film for different objective planes (P17- P19) at $\mathrm{AOI}=52^{\circ}$

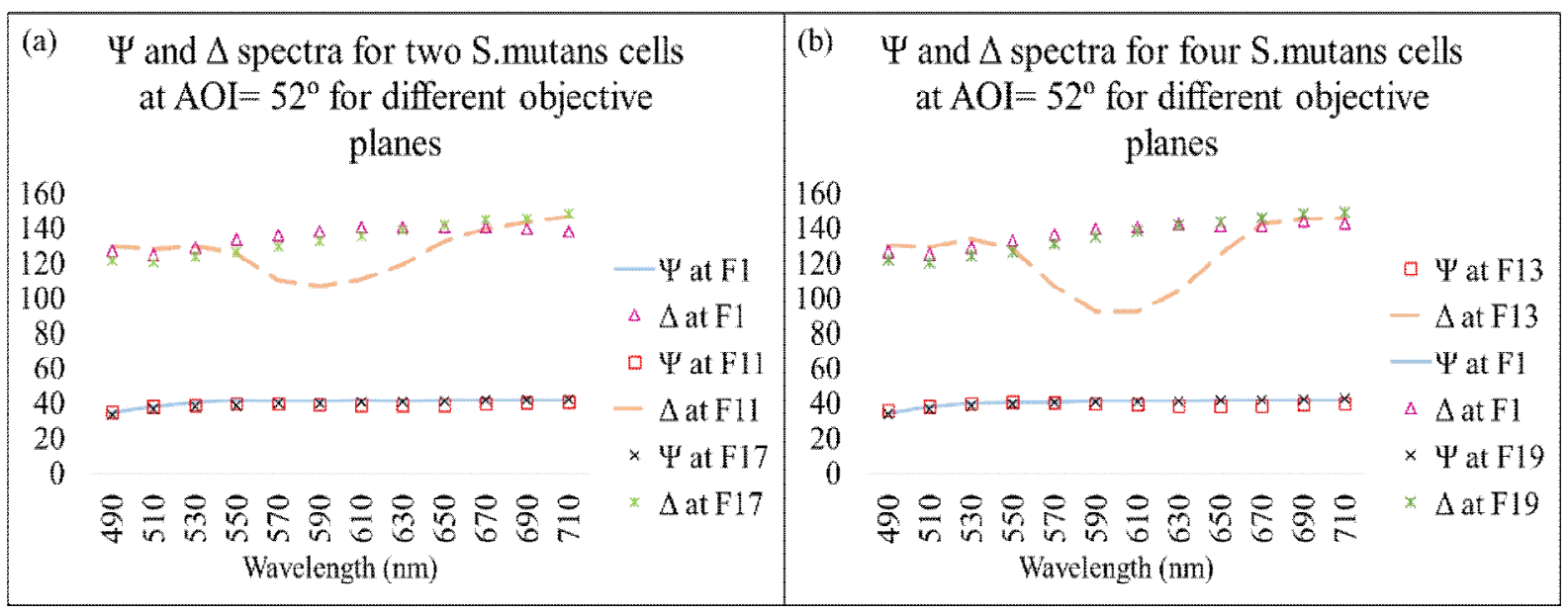

Figure 10: (a) $\Psi$ and $\Delta$ for two $S$. mutans cells of Au film obtained by MIE-RCE at different objective planes $P 1$ (in Fig. 5), $P 11$, and $P 17$ (in Fig. 6) at $A O I=52^{\circ}$, (b) $\psi$ and $\Delta$ for four $S$. mutans cells of Au film obtained by MIERCE at different objective planes $P 1, P 13$, and $P 19$ in Figure 7, Figure 8, Figure 9, respectively, at $A O I=52^{\circ}$

At $P 9$ - $P 11$ the top-view $\Delta$ images show clear signals of curved edges of the four cells and the side-view of $-\Delta$ images show a nearly connected band (see Figure 8), which indicate these object planes are close to the focal plane and the depth of focus is around $1 \mu \mathrm{m}$. Meanwhile, the CCD image displays the shape of four cells. It is also noted that the width (along the $y$-axis) of the walllike feature of side-view $-\Delta$ image correlates directly with the length of the four cells near P10.

MIE spectra for different objective planes of two and four S. mutans cells on Au film

Finally, we show the ellipsometry $\psi$ and $\Delta$ spectra of two and four $S$. mutans cells on Au film for $\lambda=490 \mathrm{~nm}-710 \mathrm{~nm}$ at $\mathrm{AOI}=52^{\circ}$ in
At $P 12$ and $P 13$, the focal plane is below the four cells, and light penetrated through the four cells and reflected at the interface between cells and the Au film, where the $\Delta$ images trace the outer profile of the four cells. Moving the stage further up to P14 and P15, distortion occurred in $\Delta$ images which are related to the diffraction pattern of the curved edges of the four cells and the signal became weak as the stage move up (further away from the focal plane). Figures 10a and Figure 10b, respectively. For the two cells, the ellipsometry spectra integrated over an area equals to $3 \mu \mathrm{m}$ (x-axis) $\times 5 \mu \mathrm{m}$ (yaxis) at three different objective planes $P 1, P 11$, and $P 17$ in Figure 5 and Figure 6, while for the four cells the ellipsometry spectra integrated over an area equals to $4 \mu \mathrm{m}$ (x-axis) $\times 9 \mu \mathrm{m}$ (y-axis) 
at three different objective planes $P 1, P 13$, and $P 19$ in Figure 7, Figure 8 and Figure 9.

The $\psi$ spectra in Figure 10a and Figure 10b for two and four $S$. mutans cells at different objective planes are essentially the same, which indicates the $\psi$ images are not sensitive to the position of the objective plane. The $\Delta$ spectra for the two cells at $P 1$ and $P 11$ match each other in the range of $\lambda=490 \mathrm{~nm}$ to $710 \mathrm{~nm}$, where the two cells are out of focus at these objective planes as shown in Figure 5 and Figure 6. For P13 (Figure 6 ) the two cells are slightly above the focal plane and benefited from the strong interference between the scattered wave and the reflected wave from the substrate, the measured $\Delta$ spectra of the two cells show different behavior in the range of $\lambda=550 \mathrm{~nm}$ to $650 \mathrm{~nm}$, but remain similar to the ones at $P 1$ and $P 17$ in the range of $\lambda=(490-540 \mathrm{~nm})$ and $(660-710 \mathrm{~nm})$. Similar behavior for the $\Delta$ spectra of four $S$. mutans cells is seen in Figure $10 \mathrm{~b}$ for the three different objective planes $P 1, P 13$, and $P 19$. These results indicate that $\Delta$ spectra are also very sensitive to the position of the objective plane and the meaningful $\Delta$ spectra of $S$. mutans cells can be obtained only at the suitable objective plane.

From these results we noticed that $\Delta$ image results exhibit high sensitivity to the morphology of the object and the position of objective plane (POP), which allows the use of $\Delta$ images in the future to determine the accurate position of the focal plane and allow quantitative assessment of biological samples with submicron features. The intensity related images $\left(l_{s}, l_{p}\right)$ show complementary information to that obtained by $\Delta$ images. $\Delta$ images show a strong spatial variation in the side view (which delineates the structure feature) at objective planes close to the focal plane, while the polarization-contrast related images ( $\Psi$ and $\mathrm{RDI}$ ) exhibit weak variation at these objective planes. The ellipsometry spectra results of two and four $S$. mutans also show the high sensitivity of $\Delta$ spectra to POP, while the $\psi$ spectra are nearly independent of the objective plane.

To get a close-up view of the $\Delta$ images, we show the 3D perspective of $\Delta$ images of two cells (at $P 10$ ) and four cells (at P13) in Figure 11a and Figure 11b.

The 3D perspective of $\Delta$ images in Figure 11a and Figure $11 \mathrm{~b}$ reveals the curved edges associated with the cells with two (four) curved edges appearing for the two (four) cells as indicated by red arrows. To give a better perspective, we have rotated the frame so that the detector arm is along the direction of the arrows. To lend further support to our analysis regarding the morphologies of the $S$. mutans cells, we show in Figure 8a an AFM topography image for a chain of four $S$. mutans cells on a glass substrate. We chose this image from a separate measurement where many AFM topography images were collected for S. mutans cells cultured for 24 hours on glass substrate.

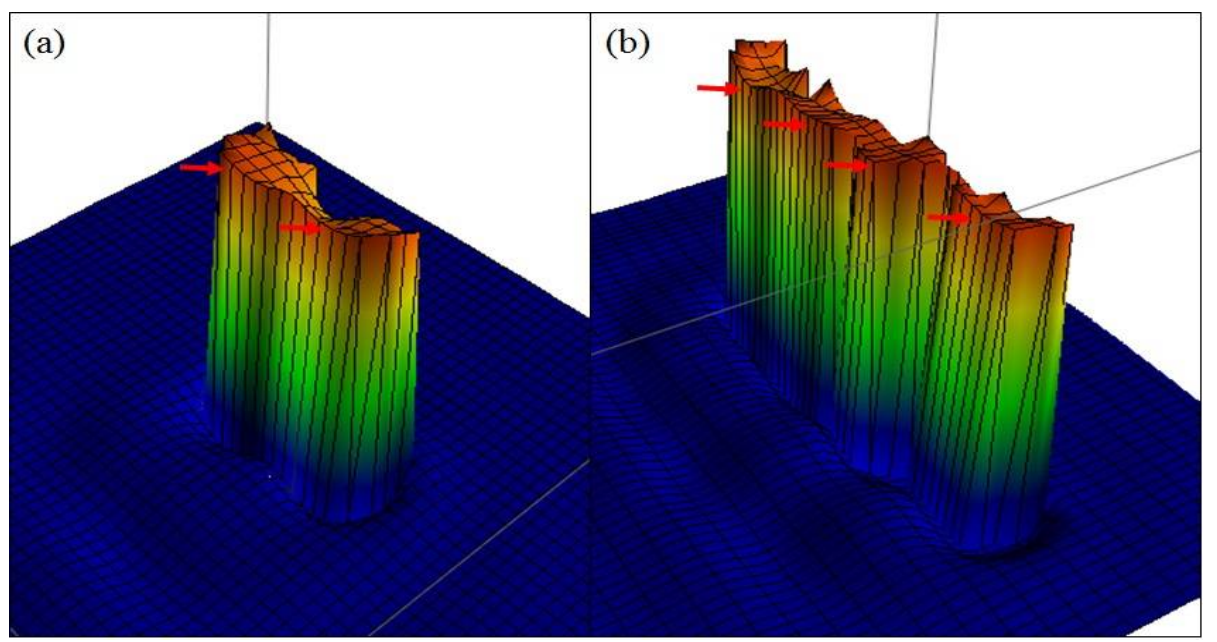

Figure11: 3D perspective of $\Delta$ images of (a) two $S$. mutans cells at $P 10$, and (b) four $S$. mutans cells at $P 13$

\section{DISCUSSION}

The findings show that the $\Delta$ images for two and four dried $S$. mutans cells exhibit big changes at different objective planes. Only at specific objective planes (close to the focal plane) do the cells features appear clearly. These objective planes are just above the focal plane, and the interference of scattered light with the reflected light from the Au film is strongest.

Trop J Pharm Res, November 2017; 16(11): 2723 

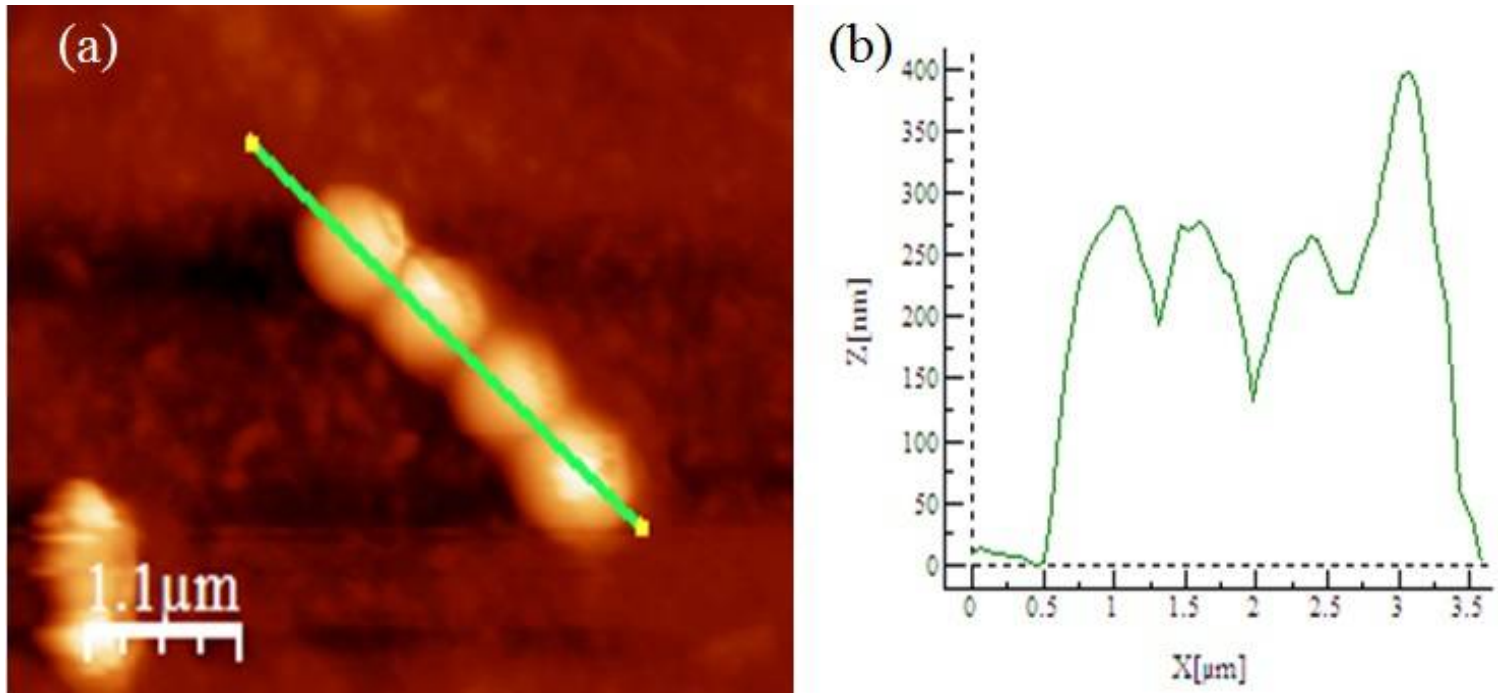

Figure 12: Four $S$. mutans cells on glass substrate after 24-h culture: (a) $5.7 \mu \mathrm{m} \times 5.7 \mu \mathrm{m}$ AFM topography image, and (b) AFM profile height of the four cells

Figure $12 b$ shows AFM height profile of the four $S$. mutans cells seen in Figure 11a. The height profile shows a size of $800 \mathrm{~nm}$ for each $S$. mutans cell, and the height of $S$. mutans cells ranges from $300 \mathrm{~nm}$ to $400 \mathrm{~nm}$. Comparing with the AFM results of $S$. mutans cells, the prediction of cell dimension based on our ellipsometry $\Delta$ images is consistent, and it demonstrates that our MIE technique is capable of identifying submicron features (in this case $S$. mutans cell with $800 \mathrm{~nm}$ size).

\section{CONCLUSION}

The results obtained in this study show the power of MIE to identify sub-micron scale features, and track the diffraction patterns at different objective planes. This study reveals that MIE is a powerful sensitive imaging technique that can be used to study biological systems, since it is non-destructive, and does not require labeling of molecules like other imaging techniques. Furthermore, by incorporating theory simulation, based on the full solution to Maxwell equation for light scattering, it will be very easy to determine the shape of the sample beyond diffraction limit.

\section{DECLARATIONS}

\section{Acknowledgement}

This work was supported, in part, by the Ministry of Science and Technology of Taiwan under contract no. MOST 104-2112-M-001-009-MY2.

\section{Conflict of Interest}

No conflict of interest associated with this work.

\section{Contribution of Authors}

The authors declare that this work was done by the authors named in this article and all liabilities pertaining to claims relating to the content of this article will be borne by them.

\section{Open Access}

This is an Open Access article that uses a funding model which does not charge readers or their institutions for access and distributed under the terms of the Creative Commons Attribution License (http://creativecommons.org/licenses/by/ 4.0) and the Budapest Open Access Initiative (http://www.budapestopenaccessinitiative.org/rea d), which permit unrestricted use, distribution, and reproduction in any medium, provided the original work is properly credited.

\section{REFERENCES}

1. KG PIPGCP-ZPZS. [https://www.physikinstrumente. com/en/products/linear-stages-and- actuators/piezostages/p-2611z-piezo-z-stage-201731/].

2. Yoshizawa Tr. Handbook of optical metrology: Principles and applications. Boca Raton: CRC Press. 2009; xiii, $p$ 730.

3. Bunshah RF. Handbook of deposition technologies for films and coatings: science, technology, and applications. 2nd edn. Materials science and process technology series Electronic materials and process technology. Park Ridge, N.J.: Noyes Publications. 1994. Xxvi; $p 861$.

4. Bubert $H$, and $H$ Jenett. Surface and thin film analysis: principles, instrumentation, applications. Weinheim: Wiley-VCH. 2002. xvii, $p 336$.

Trop J Pharm Res, November 2017; 16(11): 2724 
5. Fujiwara H. Principles of Spectroscopic Ellipsometry, in Spectroscopic Ellipsometry. John Wiley \& Sons Ltd, 2007; pp 81-146.

6. Ducheyne P. Comprehensive biomaterials. Amsterdam; Boston: Elsevier. 2011.

7. Dutta M, MS Rahman, AS Bhalla, and RY Guo. Optical and microstructural characterization of multilayer $\mathrm{Pb}$ (Zr0.52Ti0.48)O-3 thin films correlating ellipsometry and nanoscopy. J Mater Sci 2016; 51(17): 7944-7955.

8. Peled A. Photo-excited processes, diagnostics, and applications: fundamentals and advanced topics. Boston: Kluwer Academic Publishers. 2003; xv, p 370.

9. Arwin H. Spectroscopic ellipsometry and biology: recent developments and challenges. Thin Solid Films 1998; 313: 764-774.

10. Qavi AJ, AL Washburn, JY Byeon, and RC Bailey. Labelfree technologies for quantitative multiparameter biological analysis. Anal Bioanal Chem 2009; 394(1): 121-135.

11. Arwin H. Ellipsometry on thin organic layers of biological interest: characterization and applications. Thin Solid Films 2000; 377: 48-56.

12. Jones $A C$, and $M B$ Raschke. Thermal Infrared Near-Field Spectroscopy. Nano Letters 2012; 12(3): 1475-1481.

13. Lewandowska R, M Gaillet, GLe Bourdon, C Eypert, S Morel, C Naudin, and M Stchakovsky. What can Raman spectroscopy and spectroscopic ellipsometry bring for the characterisation of thin films and materials surface? Surface and Interface. Analysis 2008; 40(3-4): 588-592.

14. Khaleel MI, Y-D Chen, C-H Chien, and Y-C Chang. Sensing of Streptococcus mutans by microscopic imaging ellipsometry. J Biomedical Optic 2017; 22(5): 056005-056005.

15. Informer Technologies, I Intel (R) Array Visualizer. [cited 2017 Jan 2010]. Available from: http://intel-r-arrayvisualizer.software.informer.com/2012.2010/].
16. KG, PIPGC P-611.Z Piezo Z Stage. [cited 2017 Mar 2020]. Available from: https://www.physikinstrumente.com/en/products/linearstages-and-actuators/piezo-stages/p-2611z-piezo-zstage-201731/.].

17. Chen $Y$-D, HY Hsu, MI Khaleel, Y-C Chang, C-HWu, and $H-C W u$. Study of biological reaction in cancer cell with spectroscopic imaging ellipsometry. Proc. SPIE 9925. 2016.

18. Creative Life Science. Product category, [cited 2017 Feb 2015]. Available from: http://www.cmp-micro.com.].

19. Hu Y, J Zhang, and J Ulstrup, Interfacial electrochemical electron transfer processes in bacterial biofilm environments on $A u(111)$. Langmuir, 2010. 26(11): 9094-9103.

20. O Optrel. Ellipsometry; [cited 2017 Jan 2025]. Availble from: http://www.optrel.de/products_multiskop_ellipso metry.html.].

21. FIANIUM, WhiteLase SC series: blue \& UV enhanced supercontinuum fiber lasers. [cited 2017 Jan 2017] Available from: http://www.fianium.com/pdf/WhiteLase_ SC2400_UV_v2011.pdf.].

22. Fan JY, HX Li, and FQ Wu. A study on transmitted intensity of disturbance for air-spaced Glan-type polarizing prisms. Optics Communic 2003. 223(1-3): 1116.

23. Inc, EO Mitutoyo Infinity Corrected Long Working Distance Objectives. [cited 2017 Mar 2024]. Available from: https://www.edmundoptics.com.tw/microscopy/ infinity-correctedobjectives/Mitutoyo-Infinity-CorrectedLong-Working-Distance-Objectives/].

24. PCO pixelfly qe. [cited 2017 Jan 2020]. Available from: https://www.pco.de/sensitive-cameras/pixelfly-qe/.].

25. Woollam, JA. Vase ellipsometry. [cited 20172003 2005]. Available from: https:/www.jawoollam.com/products/ vase-ellipsometer. 